\title{
ELEMENTARY SCALING LAWS FOR THE DESIGN OF LOW AND HIGH POWER HALL EFFECT THRUSTERS
}

\section{K. Dannenmayer and S. Mazouffre}

\author{
ICARE, CNRS \\ Avenue de la Recherche Scientifique 1c, Orléans 45071, France
}

\begin{abstract}
An advanced set of scaling laws for Hall effect thrusters running with Xenon as propellant is established on the basis of the existence of an optimum atom number density that warrants a high efficiency thruster operation. A set of general relationships between macroscopic quantities, like thrust and input power, dimensions, including the channel length, the channel width and the channel mean diameter, and magnetic field strength are inferred from the main physical processes at work in a Hall thruster discharge. The "atom density constraint" of which the nature is here critically interpreted allows simplifying those relationships as it leads to a linear dependency between the channel length and mean diameter. Scaling laws which represent an essential tool for sizing up and down Hall thrusters are eventually obtained after proportionnality coefficients are determined. This last step is realized by means of a vast database that presently encompasses 33 single-stage Hall thrusters. In order to illustrate the usefulness of this new set of scaling laws, two practical applications are given and discussed. The scaling laws are first employed to calculate the dimensions and the operating parameters for a 20-kilowatt Hall thruster capable of producing $1 \mathrm{~N}$ of thrust. Such an electrical engine would permit orbit transfer of large communication satellites. Finally, the geometry of a Hall thruster is determined for tolerating $100 \mathrm{~kW}$, an interesting power level for interplanetary trips.
\end{abstract}

\section{NOMENCLATURE}

$\begin{array}{ll}B & \text { magnetic field strength } \\ C_{B} & \text { proportionality coefficient for } B \\ C_{h} & \text { proportionality coefficient for } h \\ C_{I} & \text { proportionality coefficient for } I_{d} \\ C_{T} & \text { proportionality coefficient for } T\end{array}$

This is an Open Access article distributed under the terms of the Creative Commons Attribution-Noncommercial License 3.0, which permits unrestricted use, distribution, and reproduction in any noncommercial medium, provided the original work is properly cited. 


\begin{tabular}{|c|c|}
\hline$C_{L}$ & $\begin{array}{l}\text { proportionality coefficient for } L \text { : ratio between free } \\
\text { ionization mean path and channel length }\end{array}$ \\
\hline$d, d_{\mathrm{ext}}, d_{\mathrm{int}}$ & mean, external, internal channel diameters \\
\hline$h$ & channel width \\
\hline$I_{\mathrm{d}}$ & discharge current \\
\hline$I_{\mathrm{sp}}$ & specific impulse \\
\hline $\mathrm{k}_{\mathrm{B}}$ & Boltzmann constant \\
\hline$L$ & channel length \\
\hline$m_{n}, m_{i}$ & propellant atom mass, ion mass \\
\hline$\dot{m}, \dot{m}_{i}$ & $\begin{array}{l}\text { propellant mass flow rate through the anode, ion } \\
\text { mass flow rate }\end{array}$ \\
\hline$n_{n}$ & atom number density \\
\hline$P$ & input power \\
\hline$r_{\mathrm{Le}}, r_{\mathrm{Li}}$ & electron and ion Larmor radius \\
\hline$T$ & thrust \\
\hline$T_{e}$ & electron temperature \\
\hline $\begin{array}{l}T_{\max }, T_{\text {ext }}, T_{\text {int }} \\
U_{d}\end{array}$ & $\begin{array}{l}\text { maximum, external, internal wall temperature } \\
\text { discharge voltage }\end{array}$ \\
\hline$v_{n}, v_{e}, v_{i}$ & thermal velocity of atoms, electrons, ions \\
\hline$\alpha$ & propellant conversion efficiency \\
\hline$\eta$ & thrust efficiency \\
\hline$\lambda_{i}$ & ionization mean free path \\
\hline$\nu_{c e}$ & electron gyrofrequency \\
\hline$\nu_{e n}$ & electron-atom collision frequency \\
\hline$\sigma_{i}, \sigma_{e n}$ & $\begin{array}{l}\text { cross section for ionization and electron-atom mo- } \\
\text { mentum exchange }\end{array}$ \\
\hline$\tau_{c e}$ & gyroperiod \\
\hline$\tau_{e n}$ & electron-atom collision time \\
\hline
\end{tabular}

\section{INTRODUCTION}

It is well known that chemical systems store their energy in the propellant. They are said to be "energy limited" as the chemical reactants have a fixed amount of energy per unit mass which limits the achievable exhaust velocity or specific impulse. In contrast, the rate at which energy can be supplied to the propellant is independent of the mass of propellant; so, very high power and thrust levels can be achieved. Electric propulsion systems are not energy limited. Neglecting component lifetime considerations, an arbitrarily large amount of energy can be delivered from solar arrays or a nuclear battery to a given mass of propellant so that the specific impulse can be much larger than that available from a chemical engine. Therefore, electric thrusters offer an attractive way to save propellant 
mass thanks to a much faster propellant ejection speed. Electric propulsion systems are termed "power limited" because the rate at which energy from the external source is supplied to the propellant is limited by the mass available for the power system. This has the result of limiting the thrust of the electric propulsion system for a given spacecraft mass. As a consequence electric propulsion devices tend to be low thrust-to-mass ratio - therefore, low acceleration devices. Although electric thrusters have low thrust-to-mass ratios, they can have a larger amount of total impulse in comparison with chemical rocket jets. The total impulse corresponds to the product of specific impulse and propellant mass, i. e., the total change of momentum. So, even though a chemical engine offers a high thrust-to-mass ratio, the propellant is expended in a short time at low specific impulse. On the contrary, a low thrust-to-mass ratio electric thruster can be operated for periods ranging from hours to years and build up a larger total impulse.

There are three different types of electric propulsion systems, categorized according to the method used to accelerate the propellant, as electrothermal, electrostatic, and electromagnetic. Electrothermal propulsion systems accelerate the propellant using heating. There are different subtypes: resistojets, arcjets, and inductively or radiatively heated systems. Electromagnetic propulsion uses orthogonal electric and magnetic fields to apply a Lorentz body force to ionized propellant atoms that are subsequently accelerated out of the crossed-field region. Electromagnetic propulsion techniques currently in use or being investigated include pulsed plasma thrusters and magnetplasmadynamic thrusters. Electrostatic propulsion systems accelerate the ionized propellant by means of an electric field. The main techniques are field effect electrostatic propulsion, colloidal thrusters, and gridded ion accelerators. Hall effect thrusters (HET) have been classed as both electrostatic and electromagnetic propulsion systems. An electrostatic field accelerates the ions in the propellant stream but that field is, to a large extent, produced by the actions of plasma electrons interacting with a magnetic field, giving both classes a claim to the technique.

Hall effect thrusters, also called stationary plasma thrusters or closed electron drift thrusters, are currently recognized as an attractive propulsion means for long duration missions and for maneuvers that require a large velocity increment. They are advanced propulsion devices that use an electric discharge with magnetized electrons to ionize and accelerate a propellant gas $[1,3,2]$. Due to interesting features in terms of propellant ejection speed, efficiency, thrust-to-power ratio and lifetime, HET are now employed for missions like geosynchronous communication satellite orbit correction and station keeping. Moreover, HET appear as good candidates to be used as primary propulsion engine for space probes during interplanetary journeys as demonstrated by the successful SMART-1 Moon flyby solar-powered mission of the European Space Agency [4].

In a Hall thruster, acceleration of heavy atomic ions to high velocity occurs within the core of the plasma where ions are produced, which implies the use 


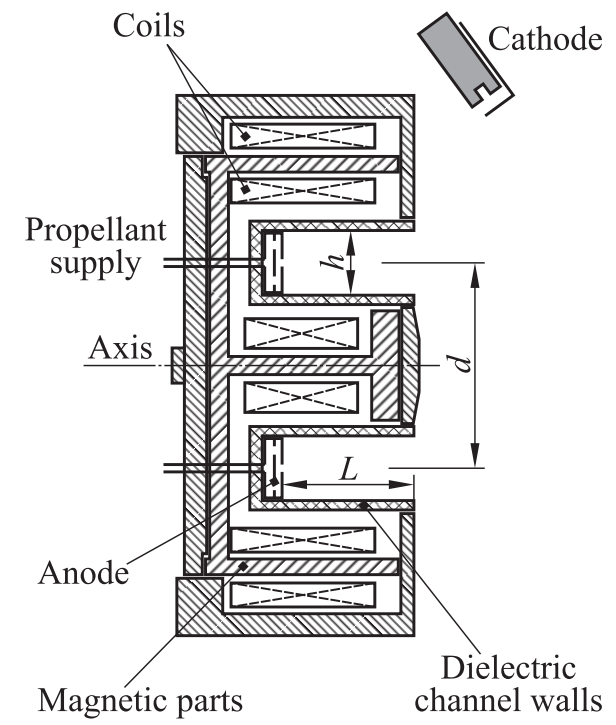

Figure 1 Schematic of a Hall thruster. The three characteristic dimensions $L, h$ and $d$ are also shown of a magnetized plasma able to sustain internal electric fields. A schematic of a HET is depicted in Fig. 1. The basic physics of a HET, therefore, consists of a magnetic barrier in a low pressure direct current (DC) discharge generated between an external hollow cathode and an anode. The anode, which also serves as gas injector, is located at the upstream end of a coaxial annular dielectric channel that confines the discharge. Xenon is generally used as propellant gas for its specific properties in terms of atomic mass and low ionization energy.

A set of solenoids provides a radially directed magnetic field $B$ of which the strength is maximum in the vicinity of the channel exhaust. The magnetic field is chosen strong enough to make the electron Larmor radius much smaller than the discharge chamber length, but weak enough not to affect ion trajectories. The electric potential drop is mostly concentrated in the final section of the channel owing to the low electron axial mobility in this restricted area. The corresponding induced local axial electric field $E$ has two main effects. First, it drives a high electron azimuthal drift — the Hall current - that is responsible for the efficient ionization of the supplied gas. Second, it accelerates ions out of the channel, which generates thrust. The ion beam is neutralized by a fraction of electrons emitted from the hollow cathode. When operating near $1.5 \mathrm{~kW}$, a HET ejects ions at $20 \mathrm{~km} / \mathrm{s}$ and generates $100 \mathrm{mN}$ of thrust with an overall efficiency of about $50 \%$.

New fields of application are nowadays envisaged for electric propulsion systems that require low and high power devices. Low power Hall thrusters $(\sim 100 \mathrm{~W})$ are well suited for drag compensation of observation satellites that operate on a low-altitude orbit in the Earth atmosphere as well as for orbit correction and orbit transfer of small platforms. The use of high power Hall thrusters $(\sim 5 \mathrm{~kW})$ for orbit raising and orbit topping maneuvers of communication satellites would offer significant benefits in terms of launch mass, payload mass, and operational life. In addition, orbit transfer of large platforms and journeys to far-off planets and asteroids with large and heavy robotic probes necessitates to build thrusters with an input power in the range of 10-100 kW. In 
view of project demand, it appears necessary to expand the operating envelope of existing HET technology to achieve the required performance level. A nontrivial question then arises: How to extrapolate the design and architecture of currently existing Hall thrusters towards different scales and different operating conditions? In other words: What are the elementary scaling laws that connect HET characteristic dimensions with operating parameters like discharge voltage, propellant mass flow rate, and magnetic field strength and with performances in terms of thrust, specific impulse, and overall efficiency?

Scaling laws that govern the physical properties, the acceleration ability, as well as the propellant and energy consumption of Hall thrusters have been extensively investigated by numerous authors since the period of development of Hall thrusters in the 1970s [1,5-7]. In spite of many years of research on this subject, the assessment of scaling laws is still a topic of great interest, with debates and controversies as various methodologies and results exist. A critical review on scaling laws and sizing methodologies developed over the past decade was made in a previous article [8]. Presented in the same contribution was an innovative method to extrapolate the Hall thruster geometry towards both low and high power ranges that avoids the utilization of elaborate computer simulation tools. The method is based on the combination of a set of scaling laws with a vast body of existing data. It considers the three characteristic thruster dimensions, i. e., the channel length, the channel width and the channel mean diameter as well as the magnetic field strength. Analytical scaling laws are built from the physical principles that govern the properties of a Hall thruster discharge. To obtain simple relations between quantities of interest, a list of simplifying but reasonable assumptions is necessary. Among others, it was assumed that quantities are steady and homogenous in the plasma, the applied potential energy is fully converted into axial kinetic energy, and all xenon ions are singly-charged. Experimental data about Hall thrusters operating with xenon were subsequently employed to accurately assess proportionality coefficients appearing in scaling laws. Besides, a thermal constraint on the channel walls was introduced to narrow down the range of possible values for thruster dimensions. Finally, the authors' sizing methodology was applied for the design of 20- and 25-kilowatt Hall thrusters, which are well-suited electric engines for orbit transfer of geosynchronous platforms as well as for interplanetary robotic space probes.

The objective of next works, of which the content is summarized in this paper, was to refine the set of scaling laws. The approach was twofold, namely: to better account for the propellant atom density constraint, which was briefly highlighted in the authors' preceding study, and to enlarge the database including new thruster performance data, especially for input power above $5 \mathrm{~kW}$. A detailed investigation of the authors' experimental data revealed the existence of an optimum atom density inside the channel that warrants high efficiency thruster operation. This "density constraint" has an immediate consequence for the thruster dimensions. The authors indeed demonstrated the "constraint" im- 
plies a mere linear relationship between the channel width and the channel mean diameter, which automatically simplifies the previously established scaling laws. In addition, proportionality coefficients that appear in the new set of scaling laws were determined by means of a database that comprises now 33 singlestage HET, instead of 23 previously. With simplified scaling laws and a greater number of performance data, the sizing procedure becomes with no doubt more accurate.

In the last section of this paper, the new scaling laws are employed to calculate the geometry and the magnetic field strength of a 20-kilowatt Hall thruster able to deliver a thrust of $1 \mathrm{~N}$. Results are then compared with the ones obtained with former laws. Furthermore, sizes for a 100-kilowatt-class Hall thruster that could find applications for cargo missions and for exploration missions to far-off planets are specified.

\section{THEORETICAL APPROACH}

A necessary first step in order to determine scaling laws for HET does consist in finding some critical parameters as well as in defining the similarity criteria based on the current knowledge and understanding of the physics of Hall thrusters. The geometry of a HET is defined by three characteristic dimensions: discharge channel length $L$, mean diameter $d=\left(d_{\text {ext }}+d_{\text {int }}\right) / 2$, and channel width $h$, as well as by a set of operating parameters such as the magnetic field strength $B$, the discharge voltage $U_{d}$, and the propellant mass flow rate $\dot{m}$.

To simplify the assessment of scaling laws, the following assumptions have been made throughout the entire paper:

- all quantities are steady in time and uniform in space;

- the electron temperature is unchanging whatever the operating conditions;

- the propellant gas has a uniform and fixed temperature all over the channel; hence, a constant propellant velocity;

- the potential energy is fully converted into kinetic energy and all ions experience the whole potential drop, of which the magnitude is $U_{d}$;

- plasma-wall interactions are taken into account through heat load to the channel walls;

- the magnetic field highest magnitude is solely considered;

- electron transport across the magnetic barrier is considered as classical: no anomalous transport is accounted for within the region of strong magnetic field [9]; 
- there are no multiply-charged ions in the plasma [10]; and

- a parallel monokinetic ion beam is produced, i. e., the plasma jet divergence is null.

To ensure a sufficient ionization of the gas, the ionization mean free path $\lambda_{i}$ must be much smaller than the channel length $L$. This is the so called MelikovMorozov criterion:

$$
\lambda_{i}=\frac{v_{n}}{n_{n} \sigma_{i} v_{e}} \ll L
$$

where $v_{n}$ is the thermal velocity of the atoms; $n_{n}$ is the atom number density; $\sigma_{i}$ is the ionization cross section; and $v_{e}$ is the thermal velocity of the electrons.

The electrons must be efficiently trapped by the magnetic field at the channel exhaust in order to ensure a good ionization and a strong electric field for acceleration of the ions. On the other hand, the ions should not be affected by the magnetic field. This leads to the following relations:

$$
\begin{aligned}
& r_{\mathrm{Le}}=\frac{m_{e} v_{e}}{e B} \ll L ; \quad r_{\mathrm{Li}}=\frac{m_{i} v_{i}}{e B} \gg L ; \\
& \frac{\tau_{e n}}{\tau_{c e}}=\frac{\nu_{c e}}{\nu_{e n}}=\frac{e B}{m_{e} n_{n} \sigma_{e n} v_{e}} \gg 1
\end{aligned}
$$

where $r_{\mathrm{L}}$ is the Larmor radius; $\tau_{e n}$ is the electron-atom collision time; $\tau_{c e}$ is the gyroperiod of the electrons; and $\sigma_{e n}$ is the electron-atom momentum exchange cross section.

Combining relations (1)-(3) and using previously cited assumptions as well as usual definitions for thrust and $I_{\mathrm{sp}}$, one finds:

$$
\begin{aligned}
T & \propto \alpha \dot{m} \sqrt{U_{d}} ; \\
T & \propto \alpha \frac{1}{L} \sqrt{U_{d}} h d, \\
I_{\mathrm{sp}} & \propto \alpha \sqrt{U_{d}}, \quad L \propto \lambda_{i} ; \\
B & \propto \frac{1}{h d} \quad \text { and } \quad B \propto \frac{1}{l} .
\end{aligned}
$$

A detailed description of the derivation of these equations is given in previous article [8]. The proportionality coefficients $C$ can be determined using a vast database, as explained further in the paper.

\section{DATABASE}

A thorough open literature search using a wide range of resources combined with data-gathering performed within the French research program on electric 
propulsion allowed the development of a large database on HET performances. The database contains information about thruster geometry as well as performances, notably the thrust $T$, the specific impulse $I_{\mathrm{sp}}$, and the efficiency $\eta$ for a series of 33 different single-stage Hall thrusters. Moreover, the database includes information about the magnetic field strength $B$, the discharge channel wall materials, and the propellant gas. The entire database covers a vast range of input power that stretches from $10 \mathrm{~W}$ up to $50 \mathrm{~kW}$, and a large collection of data points in terms of applied discharge voltage and gas mass flow rate. A broad range of thrust level is certainly covered, going from $0.4 \mathrm{mN}$ with a micro-Hall thruster up to $2.95 \mathrm{~N}$, delivered by the high-power thruster developed at NASA.

A part of the collected data in terms of thrust level is displayed in Fig. 2. For all thrusters, channel walls are made of $\mathrm{BN}_{-} \mathrm{SiO}_{2}$ and the propellant gas is xenon. The thrusters used to construct the figure are the following: a $4 \mathrm{~mm}$ in diameter micro-Hall thruster operating at 10-40 W [11], a laboratory model of the

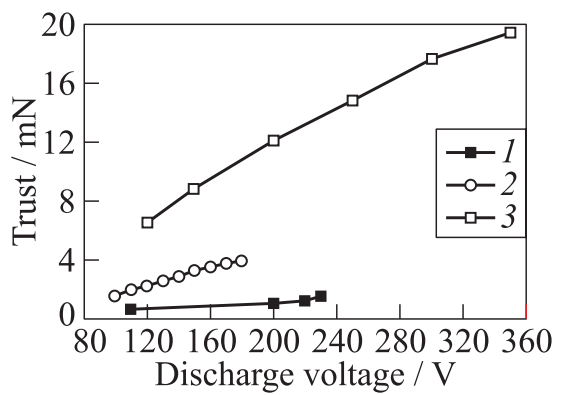

(a)

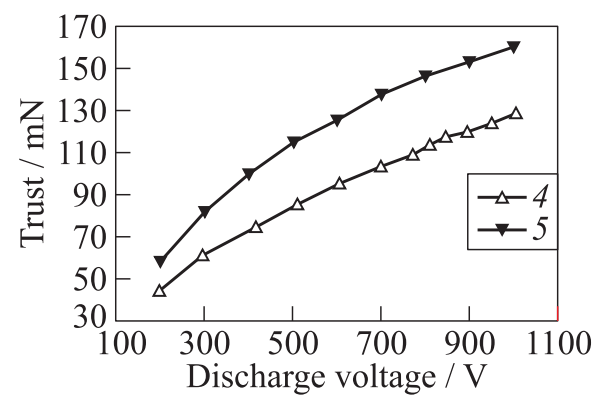

(b)

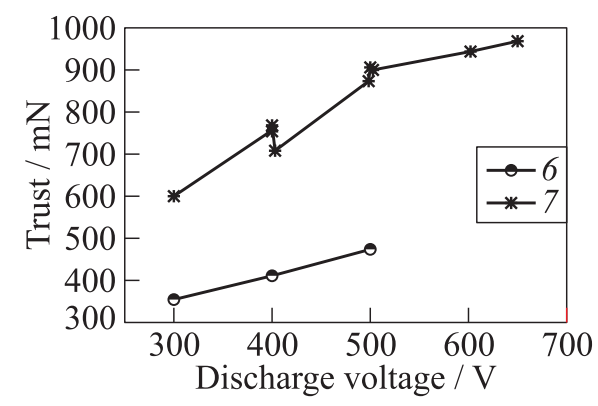

(c)

Figure 2 Thrust as a function of the discharge voltage for seven different thrusters: 1 - microthruster $(0.2 \mathrm{mg} / \mathrm{s}) ; 2-\operatorname{SPT} 20(0.472 \mathrm{mg} / \mathrm{s}) ; 3-\mathrm{SPT} 50(1.0 \mathrm{mg} / \mathrm{s}) ; 4-$ PPS ${ }^{\circledR} 1350(3.5 \mathrm{mg} / \mathrm{s}) ; 5-\mathrm{PPS}^{\circledR} \mathrm{X} 000(5 \mathrm{mg} / \mathrm{s}) ; 6-\mathrm{T} 220(19.4 \mathrm{mg} / \mathrm{s}) ;$ and 7 NASA-457M $(35.2 \mathrm{mg} / \mathrm{s})$ 
low-power SPT20 thruster [12], a SPT50 thruster manufactured by the Kurchatov Institute [12], the 1.5-kilowatt-class $\operatorname{PPS}^{\circledR} 1350$ HET developed and manufactured by SNECMA [13], the 5-kilowatt-class PPS ${ }^{\circledR} \mathrm{X} 000$ which is a laboratory version of the $\mathrm{PPS}^{\circledR} 5000$ technology demonstrator developed by SNECMA [13], the 10-kilowatt T220 designed and built by TWR and Space Power Inc. [14], as well as the 50-kilowatt-class NASA-457M thruster [15].

The propellant conversion efficiency $\alpha$ is the ratio of the ion mass flow rate to the propellant mass flow rate. Therefore, $\alpha$ is not constant but a function of the discharge volt-

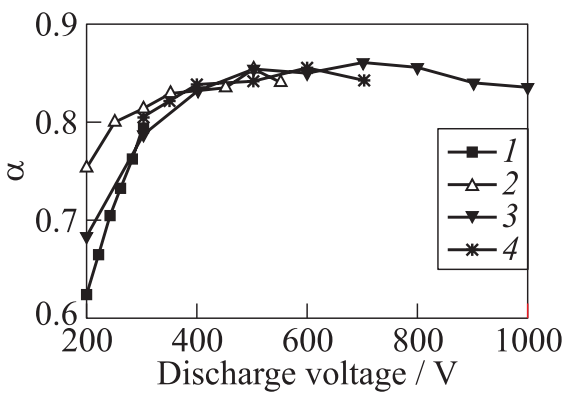

Figure 3 Propellant conversion efficiency $\alpha$ as a function of discharge voltage for three types of Hall thrusters: 1 SPT20; 2 - PPS1350; and 3-PPSX000; and 4 corresponds to values obtained for the PPS ${ }^{\circledR} \mathrm{X} 000$ thruster when the ion velocity is recorded by means of a RPA (Retarding Potential Analyzer) age and the mass flow rate. The value of $\alpha$ can be determined using the database. Figure 3 shows the calculated values of $\alpha$ for three different thrusters as a function of the applied voltage $U_{d}$ when only $\mathrm{Xe}^{+}$ions are taken into account.

This figure indicates that $\alpha$ depends both on the thruster size and on the value of $U_{d}$. For an applied voltage above $300 \mathrm{~V}$, the quantity $\alpha$ is commonly in the range of $0.8-0.9$ and thus close to the value of 0.9 given in the literature.

\section{SCALING LAWS}

It was shown in a previous paper that there is an optimum atomic number density $n_{n}$ in order to keep the physical processes at work in a Hall thrusters unchanged, to warrant a high efficiency and to limit the thermal load as well as the wall wear [8]. Using the database a value, which turns out to guarantee a satisfying operation, is: $n_{n}=1.2 \cdot 10^{19} \mathrm{~m}^{-3}$. This is also the value that is commonly found in literature [3]. When $n_{n}<n_{n, c}$, the collision events between atoms and electrons are too scarce to maintain a high ionization level. The ionization mean free path is equal $0.4 \mathrm{~mm}$ for $n_{n}=n_{n, c}$ (with $T_{n}=800 \mathrm{~K}$ and $T_{e}=20 \mathrm{eV}$ ), much below the channel length and width. For $n_{n}=10^{18} \mathrm{~m}^{-3}$, one finds $\lambda_{i}=5 \mathrm{~mm}$. The mean free path is then obviously too long. When $n_{n}>n_{n, c}$, the electron magnetic containment is weaken due to the high electronatom collision frequency inside the channel. As the transverse electron diffusion 
increases, the electric field extends and diminishes in strength, the overlap between the ionization and acceleration layers stretches out and the electron back stream through the channel outlet rises. As a consequence, the thruster efficiency drops. A basic calculation indicates that with $n_{n} \approx 10^{20} \mathrm{~m}^{-3}$, the ion and electron current magnitudes are identical at the channel exhaust. Besides, at high plasma density, ion losses to the channel walls become significant.

The existence of a critical atom number density $n_{n, c}$ leads to a surprising result: the channel width $h$ must be proportional to the channel mean diameter $d$. This relation can be verified using the database. In Fig. 4, the channel width is plotted as a function of the mean diameter for different thrusters covering a broad power range. As can be seen, the two dimensions $h$ and $d$ are proportional; this leads to the following equation:

$$
h=C_{h} d .
$$

Therefore, considering an optimum atomic number density $n_{n, c}$, one can refine the scaling laws since only two dimensions remain:

$$
\begin{aligned}
P & =C_{p} d^{2} U_{d} ; \\
T & =C_{T} d^{2} \sqrt{U_{d}} ; \\
B & =\frac{C_{B 1}}{h d} \quad \text { and } \quad B=\frac{C_{B 2}}{L} ; \\
L & =C_{L} \lambda_{i} .
\end{aligned}
$$

This set of relations can again be verified using the database. Figure 5 shows the thrust $T$ as a function of $d^{2} \sqrt{U_{d}}$ for different thrusters. The dashed line

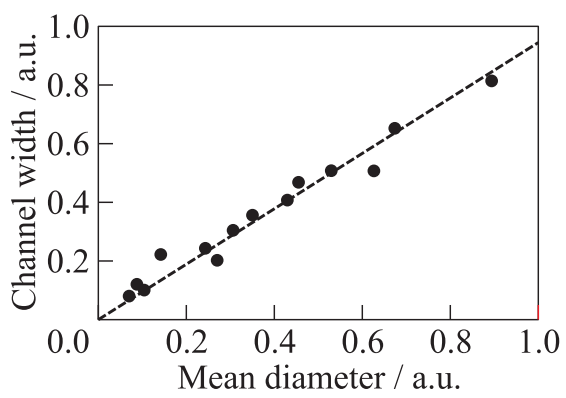

Figure 4 Channel width $h$ as a function of the channel mean diameter $d$ for a series of HET. The two dimensions $d$ and $h$ are proportional

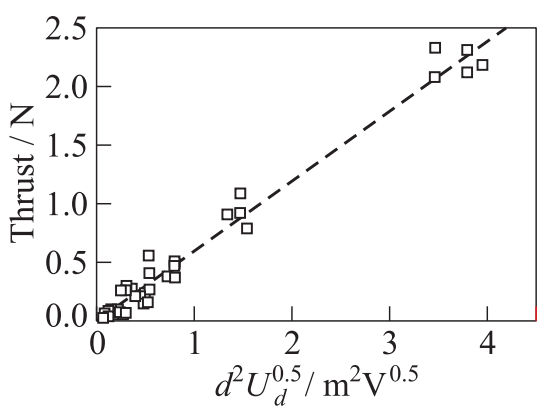

Figure 5 Thrust as a function of $d^{2} U_{d}{ }^{0.5}$ and the linear fit for different Hall effect thrusters (dashed line). For each thruster, some thrust values are chosen around the point of normal operation 
represents a linear fit through all data points. For each thruster, some values of $T$ are chosen around the point of normal operation.

One should keep in mind that Eqs. (6) and (7) are only valid when $n_{n}$ is considered to be constant and equal to the critical density $n_{n, c}$. On the contrary, the relations for the magnetic field strength given by Eq. (8) do not rely on a constant atom number density. As can be verified, when the atom density is fixed, the magnetic field strength $B$ does not depend anymore on the thruster channel dimensions $d$ and $h$. It becomes merely a function of the channel length $L$. This directly originates from the fact that the electron diffusion perpendicular to the magnetic barrier is assumed to result from electron-atom collision events. Indeed, no anomalous electron transport is considered in this work.

\section{ASSESSMENT OF PROPORTIONALITY COEFFICIENTS}

In order to assess the required thruster dimensions by way of a sizing method for a suitable input power or thrust level, it is necessary to know the proportionality coefficients of the aforementioned equations. These coefficients can be determined either empirically, using the database, or analytically from the developed scaling laws, as explained in detail in previous paper [8].

The empirically determined proportionality coefficients used for sizing a HET are naturally not given in this contribution. The $C_{P}$ and $C_{T}$ coefficients can be derived from outcomes of this study. Yet, they are available in the article previously published by Jankovsky and co-workers [16].

\section{DESIGN OF TWO HIGH POWER HALL THRUSTERS}

\subsection{0-kilowatt class Hall thruster}

High-power HET in the range of $10-30 \mathrm{~kW}$ capable of delivering a thrust level around $1 \mathrm{~N}$ with a specific impulse of about $2500 \mathrm{~s}$ are thought to be used as primary propulsion system for robotic space probes during interplanetary journeys $[17,18,19]$. Such high-power Hall thrusters may also be of interest for orbit transfer maneuvers of large satellites. Only a few high-power prototypes have been developed in the world so far and a significant research effort on this specific technology is now appearing within Europe. For this reason, the sizing method based on authors' widely applicable scaling laws in combination with the large database is employed to design a 20-kilowatt-class thruster with a thrust level of $1 \mathrm{~N}$. 
The discharge voltage is fixed to $U_{d}=500 \mathrm{~V}$ in order to limit thermal load and secondary electron emission by dielectric walls. Xenon is used as propellant gas. The channel walls are assumed to be made of $\mathrm{BN}-\mathrm{SiO}_{2}$ ceramics. The wall losses are fixed to $4 \%$ of the applied power and the ionization efficiency is fixed to 0.9 .

The scaling process that consists in determining the thruster dimensions $L$, $h$, and $d$ as well as the magnetic field strength $B$ must be carried out step by step:

1. The required mass flow rate is determined by means of Eq. (4).

2. The diameter $d$ is obtained using Eq. (7).

3. The discharge current is given by: $I_{d}=C_{I} d^{2}$.

4. The electric power reads: $P=I_{d} U_{d}$.

5. The $h$ size is found using the relationship (5).

6. The channel length $L$ is assessed using the Melikov-Morozov criterion (Eq. (9)).

7. The magnetic field strength is obtained from the relation: $B=f(L)$.

8. At least, it should be verified that the number density $n_{n}$ is close to $1.2 \cdot 10^{19}$. To do so, the proper equation is the following:

$$
\dot{m}=n_{n} m_{n} v_{n} \pi h d .
$$

The atom thermal speed is given by:

$$
v_{n}=\sqrt{\frac{8 k_{\mathrm{B}} T_{\mathrm{wall}}}{m_{n} \pi}}
$$

where $k_{\mathrm{B}}$ is the Boltzmann constant.

The channel length is the dimension that is certainly the less accurate as the proportionality coefficient $C_{L}$ exhibits a large spread. As has been shown previously [8], the ratio between $\lambda_{i}$ and $L$ is not constant but can vary considerably for different thruster geometries, gas mass flow rates, and discharge voltages. The channel length can, however, be determined using the Melikov-Morozov criterion using an averaged value for $C_{L}$. The value of $L$ can then be adjusted using the thermal constraint.

For the thermal constraint, a maximum wall temperature $T_{\max }$ is set in order to limit the thermal load on the channel walls. Knowing the thruster dimensions $L, h$, and $d$, the wall temperatures $T_{\text {ext }}$ and $T_{\text {int }}$ can be computed as a function of the input power. The process of calculating the wall temperatures 
Table 1 Dimensions, parameters, and performances evaluated from scaling laws for a 20-kilowatt Hall thruster delivering $1 \mathrm{~N}$ of thrust. The magnetic field strength $B$ is around $200 \mathrm{G}$

\begin{tabular}{|c|c|c|c|}
\hline \multicolumn{4}{|c|}{ This work } \\
\hline Dimensions & Parameters & Parameters & Performances \\
\hline $\begin{array}{l}d=270 \mathrm{~mm} \\
L=70 \mathrm{~mm}\end{array}$ & $\begin{aligned} U_{d} & =500 \mathrm{~V} \\
\dot{m} & =41.5 \mathrm{mg} / \mathrm{s} \\
I_{d} & =34.3 \mathrm{~A}\end{aligned}$ & $\begin{array}{l}P=17.2 \mathrm{~kW} \\
n_{n}=1.3 \cdot 10^{19} \mathrm{~m}^{-3} \\
T_{\text {wall }}=670 \mathrm{~K}\end{array}$ & $\begin{array}{l}T=1 \mathrm{~N} \\
I_{\mathrm{sp}}=2456 \mathrm{~s} \\
\eta=70 \%\end{array}$ \\
\hline \multicolumn{4}{|c|}{ Previous work [8] } \\
\hline Dimensions & Parameters & Parameters & Performances \\
\hline $\begin{array}{l}d=250 \mathrm{~mm} \\
L=40 \mathrm{~mm}\end{array}$ & $\begin{aligned} U_{d} & =500 \mathrm{~V} \\
\dot{m} & =41.1 \mathrm{mg} / \mathrm{s} \\
I_{d} & =33.7 \mathrm{~A}\end{aligned}$ & $\begin{array}{l}P=16.8 \mathrm{~kW} \\
n_{n}=1.12 \cdot 10^{19} \mathrm{~m}^{-3} \\
T_{\text {wall }}=880 \mathrm{~K}\end{array}$ & $\begin{array}{l}T=1 \mathrm{~N} \\
I_{\mathrm{sp}}=2760 \mathrm{~s} \\
\eta=72 \%\end{array}$ \\
\hline
\end{tabular}

can be iterative: in case that the wall temperature is above $T_{\max }$, the dimensions must be changed until the thermal constraint is satisfied. This approach is described in detail in previous paper [8]. In this work, the maximum temperature is set to $T_{\max }=900 \mathrm{~K}$. Since the values of $d$ and $h$ given by the scaling laws are quite reliable, it appears better to modify the channel length $L$ rather than the mean diameter $d$ or the channel width $h$ in case an iterative loop is necessary to satisfy the thermal constraint.

The dimensions, parameters, and performances of a 20-kilowatt-class thruster are given in Table 1 . The external wall temperatures are $T_{\text {ext }}=640 \mathrm{~K}$ and the internal wall temperature is $T_{\text {int }}=700 \mathrm{~K}$. Since $n_{n}$ is close to $n_{n, c}$, the value for the magnetic field strength $B$ is around $200 \mathrm{G}$. The results obtained using former scaling laws [8] are also given in Table 1. As one can see, the dimensions are different, especially the channel length $L$. On the contrary, the mass flow rate and performances are not much affected. The size $L$ was previously determined from the thrust being, therefore, a function of the other dimensions. Here, $L$ is found from the Melikov-Morozov criterion with a slope estimated on the basis of the enlarged database.

\subsection{0-kilowatt class Hall thruster}

The scaling methodology previously described was also employed to determine the channel sizes of a 100-kilowatt Hall thruster. Such a high-power thruster could be used as propulsion device for cargo missions to the Moon or to Mars and for exploration missions towards far-off planets and asteroids [20, 21]. For a discharge voltage $U_{d}=500 \mathrm{~V}$, one finds a mean diameter $d$ of $600 \mathrm{~mm}$. The 
thruster would then be capable of delivering a thrust of $5.8 \mathrm{~N}$ with a Xenon mass flow rate $\dot{m}=242.2 \mathrm{mg} / \mathrm{s}\left(I_{d}=200 \mathrm{~A}\right.$ and $I_{\text {Hall }} \approx 210 \mathrm{~A}$ for $\left.150 \mathrm{G}\right)$. Considering the calculated mass flow rate, $1 \mathrm{t}$ of Xenon is then consumed within $1147 \mathrm{~h}$ that means 47 days.

With such dimensions and power level, there are several constraints for thruster building and operation. Technologically, building, and assembling ceramic rings may be difficult. In like manner, the magnetic circuit architecture must provide the required field over long distances. Heat evacuation as well as Hall current induced magnetic field have to be studied. Besides, power generation is also a critical issue. In order to produce $100 \mathrm{~kW}$, one needs either very large solar panels or a nuclear battery onboard. Finally, another technical aspect to take into account is the size and the pumping capacity of the vacuum chamber for test campaigns and experimental investigations of such a large Hall thruster.

\section{CONCLUDING REMARKS AND PROSPECTS}

Starting from the existence of an optimum, or critical, atomic number density, which guarantees a high efficiency Hall thruster operation, it was possible to greatly simplify scaling laws established in a preceding study from the main physical principles that govern the thruster discharge properties. Indeed, the density constraint implies a mere linear relationship between the channel width $h$ and the channel mean diameter $d$. Moreover, the value of all proportionality coefficients inherent to the authors' scaling methodology was refined as the number of thrusters that compose the performance database was extended from $23 \mathrm{up}$ to 33. In order to illustrate the benefit of this work, the geometry and the magnetic field strength of a 20-kilowatt Hall thruster capable of delivering a thrust of $1 \mathrm{~N}$ were calculated with both "old" and "new" set of scaling laws. The field magnitude is not much affected in contrast with the channel length $L$ of which the value is modified and the error bar reduced. Performance and discharge parameters, however, stay roughly unchanged.

In the near future, the research on Hall thruster scaling laws could be orientated towards two directions. First, it is of interest to investigate the influence of simplifying assumptions upon outcomes of the sizing method by comparing an approach with weak assumptions to an approach with strong assumptions. For the weak assumption case, the electron temperature and the fraction of multiply-charged ions could be taken into account. Voltage losses and ion beam divergence could also be included. Second, the impact of the propellant choice on the thruster design is certainly worth studying. As xenon is rare and expensive, other atomic gases like krypton and argon could be used as alternative. Yet, to the best of authors' knowledge, the nature of the propellant has never been directly considered in any sizing method. 


\section{ACKNOWLEDGMENTS}

This work has been carried out in the frame of the CNRS/CNES/SNECMA/Universités joint research program 3161 entitled "Propulsion par plasma dans l'espace." It is also a part of the HiPER collaborative project financially supported in the frame of the European 7th Frame Programme (grant 218859).

\section{REFERENCES}

1. Zhurin, V. V., H.R. Kaufmann, and R.S. Robinson. 1999. Physics of closed drift thrusters. Plasma Sources Sci. Technol. 8(R1).

2. Morozov, A.I., and V.V. Savelyev. 2000. Fundamentals of stationary plasma thruster theory. In: Reviews of plasma physics. Eds. B. B Kadomtsev and V. D. Shafranov. New York: Consultant Bureau. 21.

3. Goebel, D. M., and I. Katz. 2008. Fundamentals of electric propulsion. Hoboken: Wiley.

4. Koppel, C. R., F. Marchandise, and M. Prioul. 2005. The SMART-1 electric propulsion subsystem around the moon: In flight experience. AIAA Paper No. 05-3671.

5. Ashkenazy, J., Y. Raitses, and G. Appelbaum. 1997. Low power scaling of Hall thrusters. 2nd European Spacecraft Propulsion Conference Proceedings. Noordwijk, The Netherlands: ESA Publications Division.

6. Daren, Y., D. Yongjie, and Z. Zhi. 2005. Improvement of the scaling theory of the stationary plasma thruster. J. Propul. Power 14:139.

7. Misuri, T., F. Battista, C. Barbieri, E. A. De Marco, and M. Andrenucci. 2007. High power Hall thruster design options. 30th International Electric Propulsion Conference Proceedings. Florence, Italy. IEPC Paper No. 07-311.

8. Dannenmayer, K., and S. Mazouffre. 2008. Sizing of Hall effect thrusters with input power and thrust level: An empirical approach. J. Techn. Phys. 49:3-4.

9. Koo, J. W., and I. D Boyd. 2006. Modeling of anomalous electron mobility in Hall thrusters. Phys. Plasmas 13(033501).

10. Gallimore, A.D. 2001. Near- and far-field characterization of stationary plasma thruster plumes. J. Spacecraft Rockets 38(3):441-53.

11. Ito, T., N. Gascon, W. S. Crawford, and M. A. Cappelli. 2007. Experimental characterization of a micro-Hall thruster. J. Propul. Power 23(5):1068-74.

12. Guerrini, G., C. Michaut, M. Dudeck, and M. Bacal. 1997. Parameter analysis of three small ion thrusters. 2nd European Spacecraft Propulsion Conference Proceedings. Noordwijk, The Netherlands: ESA Publications Division. 441-46.

13. Mazouffre, S., A. Lazourenko, P. Lasgorceix, M. Dudeck, S. D'Escrivan, and O. Duchemin. 2007. Exanding frontiers: Towards high power Hall effect thrusters for interplanetary journeys. 7th Symposium (International) on Launcher Technologies Proceedings. Paper No. O-25. 
14. Jankovsky, R.S., C. McLean, and J. McVey. 1999. Preliminary evaluation of a $10 \mathrm{~kW}$ Hall thruster. AIAA Paper No. 99-0456.

15. Manzella, D.H., R.S. Jankovsky, and R. R. Hofer. 2002. Laboratory model 50 kW Hall thruster. AIAA Paper No. 02-3676.

16. Jankovsky, R., S. Tverdokhlebov, and D. Manzella. 1999. High Power Hall thrusters. AIAA Paper No. 99-2949.

17. Frisbee, R.H. 2003. Advanced space propulsion for the 21st century J. Propul. Power 19(6).

18. Witzberger, K.E., and D. Manzella. 2005. Perfomance of solar electric powered deep space missions using Hall effect thruster propulsion. AIAA Paper No. 05-4268.

19. Johnson, L., R. A. Meyer, and K. Frame. 2006. In-space propulsion technologies for robotic exploration of the solar system. AIAA Paper No. 06-4687.

20. Spores, R., J. Monheiser, B. P. Dempsey, D. Wade, K. Creel, D. Jacobson, and G. Drummond. 2005. A solar electric propulsion cargo vehicule to support NASA lunar exploration programm. 29th Electric Propulsion Conference (International) Proceedings. Princeton, New Jersey. IEPC Paper No. 05-320.

21. Randolph, T. M., R. Dougherty, S. Oleson, D. Fiehler, and N. Dipprey. 2005. The Prometheus 1 spacecraft preliminary electric propulsion system design. AIAA Paper No. 05-3889. 